\title{
Development of a Urinary Lithiasis Localizer Mechanism to Couple Ultrasound and Extracorporeal Lithotripsy Equipment in Canine Model
}

\author{
Enrico Andrade, Gustavo Alarcon, Eduardo Pompeu, Archimedes Nardozza Jr, Joaquim A. \\ Claro, Valdemar Ortiz, Miguel Srougi
}

Department of Urology, Paulista School of Medicine, UNIFESP and Biotery, Faculty of Medicine, University of Sao Paulo, USP, Sao Paulo, Brazil

\begin{abstract}
Introduction: Due to the evolution of extracorporeal lithotripsy equipment (ESWL) and presently, the fact that most part of the equipment does not present ultrasound to localize urinary calculi, a system that allows adapting ultrasound equipment to ESWL equipment was developed, disposing only of fluoroscopy. Thus, this equipment was developed and was tested in urinary stones in canine models, to check its precision in relation to fluoroscopy.

Method: Seven male dogs were utilized with the introduction, in the bladder through the ureteral route, of chalkstones, with initial localization by fluoroscopy, with a further ultrasound coincidence check localization of the vesical stones, being submitted to ESWL with a 3-hour, 21 days and 60 days follow-up after the procedure.

Results: Success of localization in all animals was verified presenting elimination of stones in the first micturitions, after ESWL. No complications were verified in those animals for 60 days.

Conclusion: We verified that this equipment can lead to an update of the equipment that use only fluoroscopy, increasing in this way, their technical capacity in the treatment of urinary calculi, mainly in cases of non-radiopaque stones.
\end{abstract}

Key words: urolithiasis; bladder; lithotripsy; ultrasonography; animal models; dogs

Int Braz J Urol. 2006; 32: 583-7

\section{INTRODUCTION}

After its introduction in 1980, extracorporeal shockwave lithotripsy (ESWL) dramatically changed the direction of the treatment of urolithiasis.

Extracorporeal lithotripsy is a procedure in which urinary tract stones are pulverized in small fragments through shockwaves. Those fragments can be spontaneously eliminated.

This non-invasive procedure allows patients to be treated without the need for a surgical intervention or an endoscopic procedure.
All ESWL machines consist of the following elements: 1) a shockwave generator, 2) a shockwave focusing system, 3) a urinary stone imaging or localization system, 4) a patient coupling mechanism $(1,2)$.

Shockwave generator - All generators are based on the geometric principle of an ellipse. Shockwaves are created in the first focal point of the ellipsoid (F1 - at the ellipse center), are directed to the second focal point (F2), inside the patient. The focal zone at the F2 area, is the place where there is a maximum concentration of the shockwaves. There are three types of generators to produce shockwaves: electrohydraulic, piezoelectric and electromagnetic (Figure-1). 


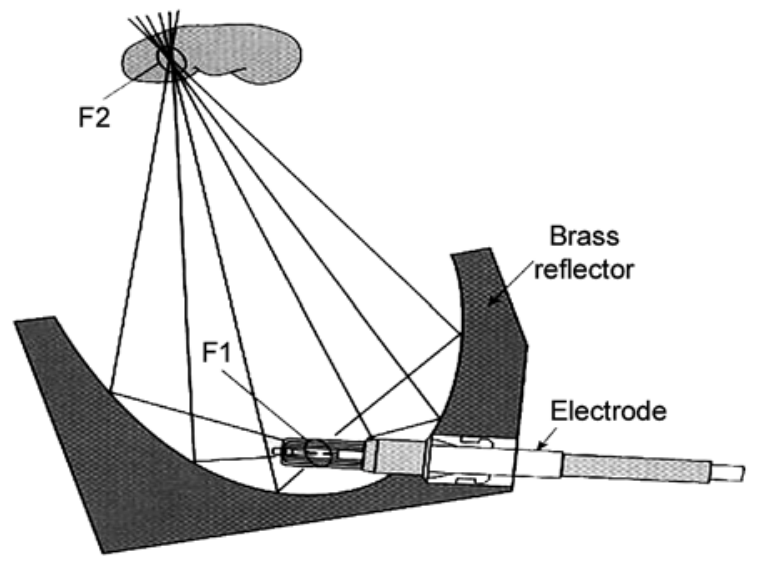

Figure 1-Shockwave focus system.

Shockwave focusing system - All the machines require a focusing system to align, direct and concentrate the energy produced at point F2 (at the stone).

Urinary stone imaging or localization system - Visualization and localization of urinary stones are used to position the stone at F2 point. Fluoroscopy and ultrasound can be used to localize the stone.

Fluoroscopy advantages are stone visualization in all the urinary tract, familiarity and ability of the urologist with the technique, possibility of using ionized contrasts and checking of anatomic details. Disadvantages are utilization of ionizing radiation, visualization of radiotransparent stones and high cost of the equipment, installation and maintenance.

Ultrasonography was initially developed to help multifunctional lithotripsy equipment where both urinary and bile stones were treated. Presently, it is used in different equipment for its lower cost and easy maintenance, when compared to those that use fluoroscopy. Advantages are visualization of radiopaque and radiotransparent stones, real time monitoring of the procedure without ionizing radiation. Despite those big advantages, there are important disadvantages: ability of the operator with the ultrasound and stones in urinary tract mid and lower ureter.

In truth, large medical centers possess new equipments that have both localization technologies such as ultrasound and fluoroscopy, despite the higher cost for the acquisition of such equipment, the option for fluoroscopy is that it can be used in other urologic endoscopic procedures. Also, there use to be a disadvantage since there was no training of the urologist for the use of ultrasound. That is not the case nowadays, since the majority of the urologists are entirely familiar with ultrasound (3-5).

Thus, the development of this equipment aims at the modernization of already existing lithotripsy equipment in use such as Breakstone, Macstone, Medstone, Econolith and Lithomax; and they can be adapted to any $7.5 \mathrm{MHz}$ probe of the most different ultrasound equipment models, either convex or linear, together with fluoroscopy of the original equipment. Such equipment would be updated and become more competitive with new equipment that holds both localization and calculation technologies.

\section{MATERIALS AND METHODS}

A mechanized system was developed for the ultrasound 7.5 MHz linear probe Hitachi EUB300 ${ }^{\circledR}$, to the lithotripsy equipment, with appropriate angulation so that the ultrasound probe keep a wave emission line passing through the F2 point. With mathematical and geometric formulas, the distance from the probe to the $\mathrm{F} 2$ point was calculated and thus, the ultrasound equipment was gauged to focus the equipment's virtual focal point, with a diameter of 5 $\mathrm{mm}$ (the ultrasound equipment focal point is standardized in $5 \mathrm{~mm}$ ), as already being the $\mathrm{F} 2$ point.

This system consists of 3 parts: 1) ESWL machine fixing system; 2) angulation and distance system of the F2 focal point and 3) ultrasound probe adapting and fixing system (Figure-2).

Thus, through the animal model with 7 male breadless medium size dogs supplied by the Biotery of the Faculty of Medicine of São Paulo. Anesthetized they were submitted to two $10 \mathrm{~mm}$ diameter cylindrical stones, chalk - dehydrated calcarean gipste in the bladder by ureteral catheterization, to deposit intravesical stones, all animals have received doses of prophylactic antibiotic therapy, according to the vet's guidance (6-8). 


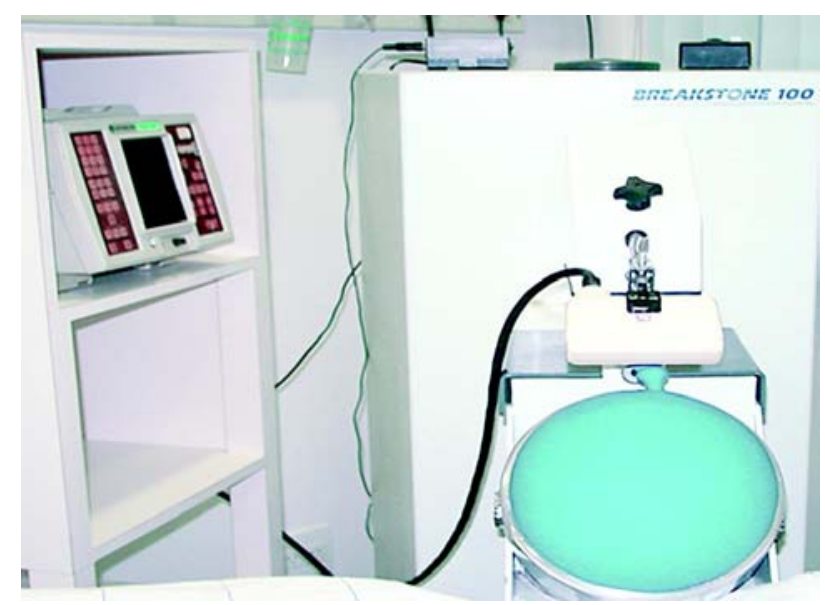

Figure 2 - Ultrasound probe adaptation and fixing system.

Initially the ESWL equipment (Breakstone, Inc) was adjusted to focus through the X-RAY fluoroscopy system (original from the lithotripsy equipment) and afterwards, the ultrasound probe was brought near the animal to verify the correspondence of the focus (stone).

After being focused the extracorporeal lithotripsy was performed for the fragmentation of those stones with $12 \mathrm{Kv}$ energy, 2000 shoots and real-time follow-up of calculi fragmentation, 3 hours after the procedure and 21 days after with the new vesical and urethral ultrasound. We have also observed urethral obstruction in the animals, by fragment of the stones that could obstruct the urethra at the point of the canine urethra where the penile canine bone is located.

\section{RESULTS}

Mean dog weight was $13.64 \mathrm{~kg}$. The procedure with the introduction of the stones and the lithotripsy had a mean duration of 72 minutes, with the performance of 2000 shoots per animal. Fragmentation of all stones and the elimination of the stones in the first micturitions of the animals were obtained, being confirmed with vesical ultrasound in the third hour after the end of the procedure (Figures-3 and 4). New ultrasounds were taken after 21 days to confirm the complete elimination of fragments.
Those animals were clinically followed for 60 days and no complications occurred such as urinary obstruction or infection (Table-1).

\section{COMMENTS}

Presently the efficiency of new extracorporeal lithotripsy machines have been little improved in relation to the first ESWL machine (HM3), thus,

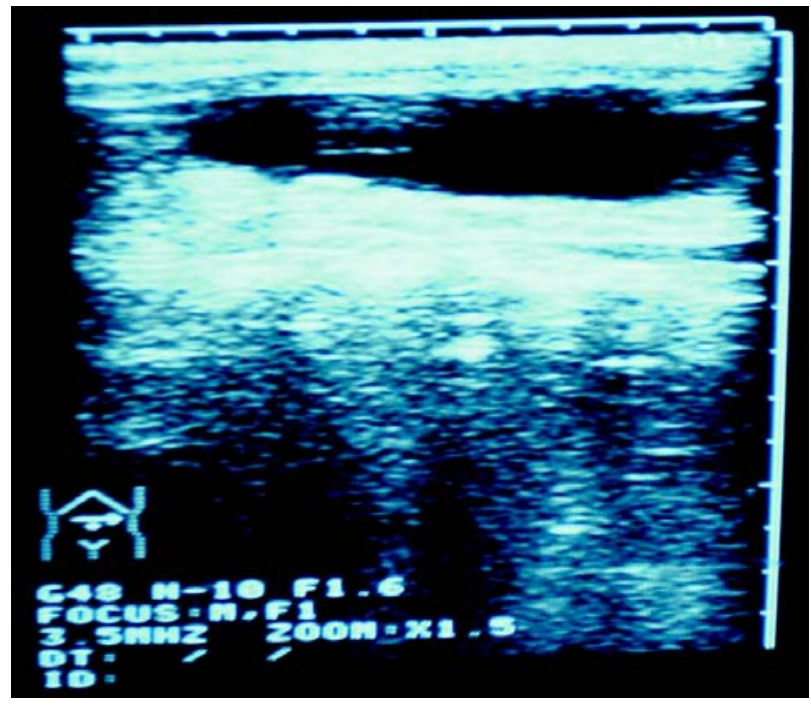

Figure 3 - Canine vesical stone, pre extracorporeal lithotripsy.

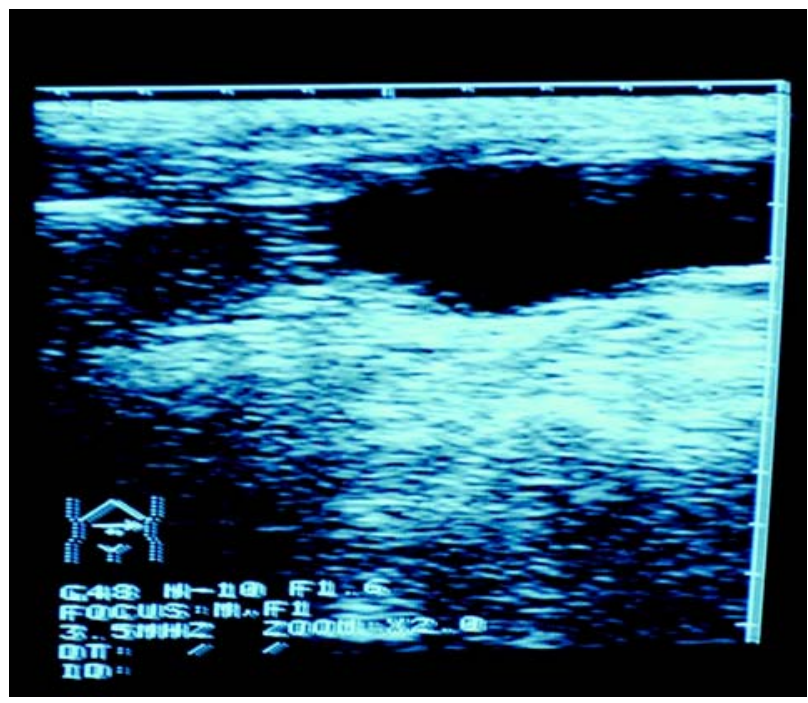

Figure 4-Canine vesical stone, post extracorporeal lithotripsy. 
Table 1 - List of animals with localization, fragmentation and complications.

\begin{tabular}{lcccc}
\hline Dogs & Weight $(\mathrm{Kg})$ & $\begin{array}{c}\text { Coinciding Localization } \\
\text { X-ray and Ultrasound }\end{array}$ & Fragmentation & Complications \\
\hline 1 & 12.0 & yes & yes & no \\
2 & 14.3 & yes & yes & no \\
3 & 11.8 & yes & yes & no \\
4 & 14.1 & yes & yes & no \\
5 & 12.7 & yes & yes & no \\
6 & 15.9 & yes & yes & yes \\
7 & 14.7 & yes & & \\
\hline
\end{tabular}

the improvement of the fragmentation method is due to the technical improvement of stone localization by ultrasound. By using the ultrasound, renal stones can be more precisely identified, mainly when some indexes are taken into consideration: 1) Stone size; 2) Radiotransparence; 3 ) Need of use of a radiological contrast; 4) Cost of the equipment and maintenance.

However, mid and inferior ureter calculi are better treated with endoscopic procedures and many calculi are not directed to the ESWL procedure for they cannot be visualized by the X-ray in the kidneys, due to their size. Thus, we can increase the percentage of those patients that are not treated by ESWL and reduce the number of risks of growth of those stones or the spontaneous elimination of larger stones that might substitute the ureter and provoke the need of an urgent endoscopic surgery.

Presently, many urologists have acquired training and have improved urologic ultrasound technique, and that is an advantage to the method of localization by ultrasonography.

Verifying the financial factor, there are great advantages, such as reduction of operational costs of approximately $60 \%$ due to various factors: 1 ) modernization of lithotripsy equipment without the need to change old models for new ones; 2) economy with radiation protection for procedure rooms; 3 ) increase of the number of cases due to radiotransparent stones; 4) decrease of costs with drugs for the contrast of the urinary tract; 5) reduction of the electric energy of fluoroscopy.

\section{CONCLUSION}

In this way, the modernization of the equipment would lead to a technical improvement and decrease of operational costs of the equipment, since initially there would be a decrease with the maintenance of fluoroscopy equipment, without the need of changing the lithotripsy equipment for a more recent model and there would be only the need to install this equipment to adapt an ultrasound.

\section{CONFLICT OF INTEREST}

None declared.

\section{REFERENCES}

1. Neisius D, Moll V: Renal ultrasonography in the management of calculus disease. Urol Clin North Am. 1989; 16: 829-40.

2. Block G, Adams LG, Widmer WR, Lingeman JE: Use of extracorporeal shock wave lithotripsy for treatment of nephrolithiasis and ureterolithiasis in five dogs. $\mathbf{J}$ Am Vet Med Assoc. 1996; 208: 531-6.

3. Jaeger P, Redha F, Marquardt K, Uhlschmid G, Hauri D: Morphological and functional changes in canine kidneys following extracorporeal shock-wave treatment. Urol Int. 1995; 54: 48-58.

4. Grasso M, Loisides P, Beaghler M, Bagley D: Treatment of urinary calculi in a porcine and canine 
model using the Browne Pneumatic Impactor. Urology. 1994; 44: 937-41.

5. Rassweiler J, Kohrmann KU, Back W, Frohner S, Raab M, Weber A, et al.: Experimental basis of shockwaveinduced renal trauma in the model of the canine kidney. World J Urol. 1993; 11: 43-53.

6. Iimori H, Senjyu M, Sugimoto T, Sugimura K, Yamamoto K, Kishimoto T, et al.: Side effects of extracorporeal shock-wave exposure on the kidney in the dogs. Nippon Hinyokika Gakkai Zasshi. 1990; 81: 400-7.
7. Paterson RF, Lingeman JE, Evan AP, Connors BA, Williams JC Jr, McAteer JA: Percutaneous stone implantation in the pig kidney: a new animal model for lithotripsy research. J Endourol. 2002; 16: 5437.

8. Houston DM, Moore AE, Favrin MG, Hoff B: Canine urolithiasis: a look at over 16000 urolith submissions to the Canadian Veterinary Urolith Centre from February 1998 to April 2003. Can Vet J. 2004; 45: 225-30.

\section{Correspondence address:}

Dr. Enrico Andrade

Rua Tijuco Preto, 845 / 42

São Paulo, SP, 03316-000, Brazil

Fax: + $55116197-0317$

E-mail: enricoandrade@uol.com.br 